\title{
Getting Your Research Out There: Open Access \& More
}

\author{
Lori A. Setton, ${ }^{1}$ Frank C. Yin, ${ }^{2}$ Susan S. Margulies, ${ }^{3}$ Shelly E. Sakiyama-Elbert, ${ }^{2}$ \\ and Doug BeIzer ${ }^{4}$ \\ ${ }^{1}$ Department of Biomedical Engineering \& Orthopaedic Surgery, Duke University, Durham, NC, USA; ${ }^{2}$ Department of \\ Biomedical Engineering, Washington University in St. Louis, St. Louis, MO, USA; ${ }^{3}$ Department of Bioengineering, University \\ of Pennsylvania, Philadelphia, PA, USA; and ${ }^{4}$ Biomedical Engineering Society, Landover, MD, USA
}

Publications in archival journals serve an important purpose in the life of a professional organization such as the Biomedical Engineering Society. BMES journals offer a venue for novel research to be vetted and reviewed by respected biomedical engineers who frequently hold positions of significance in industry or academia. This rigorous process of peer-review in a BMES journal is an oft-sought mark of support for those engineers seeking promotion, advancement or recognition amongst their peers. While numbers of citations of a published article remain the greatest recognition of scholarly work, there is growing interest in article-based metrics such as "numbers of downloads" for a specific article, recognition as the "most highly downloaded paper" in a specified period, or post-publication reviews and commentary. Springer, the publisher for all BMES journals, currently lists the number of electronic downloads for the five most heavily downloaded articles summed over a 7, 30 or 90 day period (go to www.springer.com and search for Journal name of interest). This is not only a useful measure of real-time demand for material in all BMES journals, but also an increasingly important measure of interest in individual research articles. This metric helps editors to identify hot topic areas and shifting foci and also helps BME departments to highlight and identify exciting work coming from their institutions.

Authors who publish in BMES journals have other ways to increase the visibility of their scholarship. As the publisher for all BMES journals, Springer transfers copyright to BMES in an arrangement that allows you to self-archive an author-created version of your article on your own website and/or in your institutional repository. This agreement designates Springer as a "green open access" publisher, following a classification scheme documented by the Romeo project (http://www. sherpa.ac.uk/romeo). Authors can learn more about

Address correspondence to Lori A. Setton, Department of Biomedical Engineering \& Orthopaedic Surgery, Duke University, Durham, NC, USA. Electronic mail: setton@duke.edu self-archiving and publisher copyright transfer policies for all publishers through Romeo at the indicated website. Posting your article at your institutional repository or own website can greatly raise visibility for your work immediately through the high visibility of your institution in Google and other search engines, without waiting for the fully formatted copy to be published in the BMES journals. Many US institutions have adopted this practice (see the Registry of Open Access Repositories (ROAR), http://roar.eprints.org/).

Open access publication of your article in BMES journals gets your work into the public domain immediately, ensures you the greatest possible early audience for your research, and provides access for interested news sources and scientists internationally. At this time, designation of an article in a BMES journal as Open Access is available, however, only under the "Springer Open Choice" option-for a fee paid to Springer. In addition, select articles identified for press release through the BMES national office are freely available for a limited time through special arrangements that we have negotiated with Springer on a case-by-case basis.

Still, the interest in increasing public access to scholarly literature keeps growing. The need to publicly disseminate federally-funded scholarship is wellknown to authors whose research is supported by the NIH in the USA, embodied in the NIH Public Access Policy requiring that funded researchers make their papers freely available at Pubmed Central within 12 months of publication. Many other federal governments or private foundations mandate or strongly recommend some type of public access after a prescribed embargo period, including the aggregate research councils of the United Kingdom (RCUK), the Wellcome Trust (UK), the aggregate government bodies of the European Union member states under the Open Access Pilot in FP7 (see OpenAire), and the National Health and Medical Research Council (NHMRC, Australia). A summary of the mandates for 
open access publishing are available for all international funding agencies through the JULIET database available at http://www.sherpa.ac.uk/juliet/. Springer assists compliance with these mandates through a newly developed email survey that inquires about sponsored research support at the time of article acceptance, and offers a choice of public repository (e.g., NIH Pubmed Central or UK Pubmed Central). In this manner, authors in Springer journals will soon enjoy the benefit of automatically getting your article uploaded into the Pubmed Central database with the assignation of a tracking ID and grant codes.

In addition to the arrangements for public sharing of knowledge described above, alternate models for open access and public dissemination of author-generated scholarship are much discussed in the scientific community and at BMES. With recent attempts to revoke the NIH Public Access Policy in the USA through the now-defunct Research Works Act, as well as the widely publicized boycotts of publishers supporting this legislation, the movement to support and mandate open access and shorter embargo periods is only increasing in strength. Notable developments include new proposed legislation that would at a minimum, reduce the embargo period for deposition in a public repository to 6 months in the USA (Federal Research Public Access Act or FRPAA), and the introduction of new openaccess journals paid for or supported by private foundations. One notable example is a new journal fully supported by the Wellcome Trust, Max Planck Institute and Howard Hughes Medical Institute who had originally been at the forefront of advocating open access publication for their sponsored research findings. A very large number of institutions already encourage their employees to submit articles for publication in fully open-access journals by paying open access fees (see the Compact for Open Access Publishing Equity, COPE or http://www.oacompact.org/). Harvard's Library Advisory Council took a bold step recently when they not only supported Harvard's participation in COPE, but also recommended that faculty on the editorial board of a journal consider resigning if they determine the journal content can't be published as open access. While these recommendations from a library council are bold and not clearly supported by the broader institution, the notice has served to remind us that library subscription fees for paid scientific content are higher than is sustainable for many institutions. It is not surprising that Harvard has sought to recruit their faculty base in raising the issue of open access with professional organizations and peers.

As a professional organization, BMES must be committed to publishing the highest-quality scholarship generated by biomedical engineers world-wide through a rigorous peer-review process of the most highly regarded standards. While the smaller size and financial base of BMES as a professional organization does not enable the subsidizing of open access fees for publishing in BMES journals at the current time, we are working with Springer to ensure that authors are provided all available archiving opportunities in public domain and institutional repositories. In this regard, Springer is working with its professional organizations in a manner that is not enjoyed with all publishers. At the same time, the BMES is exploring the possibility that in the future fully open-access articles could be published in a new journal, or within existing BMES publications. The BMES Publications Board held a strategic planning meeting in 2011 with input from other professional organizations that have explored or advanced the open access publication model within their organizational infrastructure. All participants recognize that publications are an important source of revenue to all professional organizations, although possibly of diminishing importance as subscription revenues may decrease in the future. The central issue is that reducing the costs for publishing scientific scholarship is critical to long-term publication success. The vitality of any publications model for a professional society may depend upon alternate revenue sources made available to authors, or to the professional organization itself. In this regard, the BMES membership overwhelmingly supported a new proposal that articles in all BMES publications be accessible in electronic format only - a change that will be implemented over the next 2 years and can be expected to reduce costs associated with printing and mailing publications.

Highlighting research articles and improving access is a priority for BMES journals in our relationship with the publisher, Springer, as we seek to bring your work into the public eye and to demonstrate the global impact and importance of biomedical engineering activities. 